Amgen Inc., G. Kricorian Shareholder of: Amgen Inc., Employee of: Amgen Inc., W. Hua Employee of: Corrona, LLC, N. Accortt: None declared DOI: 10.1136/annrheumdis-2018-eular.1509

\section{FRI0113 SWITCHING TO ANTI-IL-6 BIOLOGICS AFTER ANTI-TNF THERAPY IN CHILDREN WITH JIA}

E. Alexeeva ${ }^{1,2}$, T. Dvoryakovskaya ${ }^{1,2}$, M. Soloshenko ${ }^{1}$, R. Denisova ${ }^{1}$, K. Isaeva ${ }^{1}$, A. Mamutova', V. Gladkikh ${ }^{3,4}$, A. Moskalev ${ }^{3,4}$. ${ }^{1}$ National Medical Research Center for Children's Health; ${ }^{2}$ Sechenov First Moscow State Medical University (Sechenov University), Moscow, ${ }^{3} E O L$ Labs Itd, Department of Biostatistics; ${ }^{4}$ Institute of Computational Mathematics and Mathematical Geophysics SB RAS, Novosibirsk, Russian Federation

Background: Development of biologics gives rise to novel classes of drugs offering more options for treating children with primary or secondary failure of antiTNF therapy. However, the question of whether or not previous exposure to bio logic therapy and the number of previously administered biologics influence the efficacy of current treatment still needs to be solved.

Objectives: To compare tocilizumab efficacy in biologics-naïve and biologicsswitched patients with JIA.

Methods: Comparative analysis involved patients who had initiated TOC treatment at the National Medical Research Centre of Children's Health (Moscow) depending on previous history of biologics therapy. Treatment efficacy was evaluated according to the dynamics of clinical and laboratory signs using the ACR Pedi criteria. The Wallace criteria were used to evaluate whether or not remission had been achieved

Results: Thirty-two patients were biologics-naïve and 43 patients switched to TOC were previously treated with ETA $(n=10)$, ADA $(n=34)$, certolizumab $(n=2)$, and infliximab $(n=1)$. Children in the biologics-naïve group differed from the switchers in a number of important baseline parameters: shorter disease duration (2.13 [1.25:5.34] and 7.42 [3:10.75] years, respectively; $p<0.001)$ and lower arthritis severity indices (the number of joints affected, the CHAQ and JADAS scores). Therapy with TOC in children was found very effective. The CHAQ and JADAS disease activity scores, the CRP and ESR laboratory values, morning stiffness duration, and the VAS score (assessed by both patient and physician), and the number of affected joints (swollen or painful joints, joints with the limited range of motion and with active arthritis) significantly decreased after 4 week therapy in all patients $(p<0.01)$. The percentages of biologics-naïve patients and switchers who achieved ACR90 after the first 12 months of therapy were $31.25 \%$ and $25.6 \%$, respectively $(p=0.613)$. A smaller percentage of children achieved stable remission: $4.65 \%$ of switchers and $6.25 \%$ of biologics-naïve patients ( $p>0.999)$.

Conclusions: Tocilizumab therapy is highly efficient both as the first and subsequent biologic agent. Children with history of therapy with at least one biologic agent have lower chances for achieving remission during the first 12 months of therapy. However, this difference is most likely caused by the longer and more severe arthritis course in children allocated to the group of biologics-switched patients compared to biologics-naïve ones. Further matched large-cohort study is needed to identify predictors of response to therapy.

Disclosure of Interest: E. Alexeeva: None declared, T. Dvoryakovskaya Grant/ research support from: Roche, Pfizer, M. Soloshenko: None declared, R. Denisova: None declared, K. Isaeva: None declared, A. Mamutova: None declared, V. Gladkikh: None declared, A. Moskalev: None declared DOI: 10.1136/annrheumdis-2018-eular.6526

\section{FRI0114 EFFICACY AND RETENTION RATE OF CERTOLIZUMAB PEGOL IN RHEUMATOID ARTHRITIS: DATA FROM A LARGE REAL-LIFE MULTICENTRE RETROSPECTIVE COHORT}

E.G. Favalli ${ }^{1}$, A. Becciolini ${ }^{1}$, P. Sarzi Puttini ${ }^{2}$, F. Atzeni ${ }^{3}$, R. Bortolotti ${ }^{4}$, S. Monti ${ }^{5}$, C. Lomater ${ }^{6}$, R. Gorla ${ }^{7}$, C. Bazzani ${ }^{7}$, R. Caporali ${ }^{5}$, on behalf of on behalf of LORHEN group. ${ }^{1}$ Department of Rheumatology, Gaetano Pini Institute; ${ }^{2}$ Rheumatology Unit, University Hospital L. Sacco, Milano; ${ }^{3}$ Rheumatology, University of Messina, Messina; ${ }^{4}$ Rheumatology Unit, Santa Chiara Hospital, Trento; ${ }^{5}$ Rheumatology, University of Pavia, IRCCS Policlinico San Matteo Foundation, Pavia; ${ }^{6}$ Rheumatology, Ospedale Mauriziano, Torino; ${ }^{7}$ Rheumatology Unit, Spedali Civili, Brescia, Italy

Background: Even though certolizumab pegol (CZP) has been licensed for the treatment of rheumatoid arthritis (RA) since longtime, observational data in a reallife setting are still lacking.
Objectives: To retrospectively evaluate the use of CZP in a multicentric observational cohort of Northern Italy (the LORHEN registry), calculating both clinical response and retention rate. To explore the effectiveness of CZP in childbearing age female.

Methods: Data were retrospectively extracted from the LORHEN registry which includes all RA patients treated with CZP as first or second-line biologic agent between December 2010 and April 2017. The 2 year clinical response was evaluated as EULAR response and proportion of patients achieving Disease Activity Score 28 (DAS28-ESR) remission. The 5 year retention rate was calculated by Kaplan-Meier method. Cox proportional hazard models were developed to examine potential predictors of CZP persistence, including sex, line of CZP treatment (naïve vs switchers), childbearing age, and concomitant MTX as categorical variables, whereas age, disease duration, and baseline DAS28-ESR as continuous variables.

Results: The overall study population included 242 RA patients $(78.9 \%$ female; mean [ \pm standard deviation, SD] age $54.2 \pm 13.8$ years; mean disease duration 10 \pm 13.1 years; baseline DAS28-ESR $4.58 \pm 1.39$ ), who received CZP as first- $(64 \%)$ or second-line (36\%) biologic agent, as monotherapy $(40.9 \%)$ or in combination with methotrexate (MTX, 59.1\%). Two-year EULAR good +moderate response and remission rates were similar in first- and second-line patients $(66 \%$ vs $60.7 \%$ $[p=0.65]$ and $39.6 \%$ vs $32.1 \%[p=0.52]$, respectively). The overall 5 year retention rate was $42.5 \%$, with no difference between first- and second-line therapy $43.5 \%$ vs $40.5 \%$, respectively; $p=0.98$ ), but with a clear trend in favour of childbearing subpopulation versus older women $(62.8 \%$ vs $32.3 \%$, respectively; $p=0.07)$. Concomitant MTX was a predictor of CZP persistence (Hazard Ratio [HR] 1.79, 95\% confidence interval [95\% Cl] 1.08-2.95; $p=0.02)$, whereas sex (HR 1.35, 95\% C $0.71-2.54, \mathrm{p}=0.35)$, age ( $\mathrm{HR} 1.01,95 \% \mathrm{Cl} 0.99-1.03 ; \mathrm{p}=0.14)$, mean disease duration (HR 0.99, 95\% Cl 0.97-1.02; $\mathrm{p}=0.87$ ), and baseline DAS28-ESR (HR $1.15,95 \% \mathrm{Cl} 0.96-1.38 ; \mathrm{p}=0.12$ ) were not associated with CZP retention rate. The most frequent reason for discontinuation was inefficacy $(60 \%)$, whereas only $21 \%$ of patients stopped the drug because of adverse events.

Conclusions: In our real-life experience, CZP showed a very good clinical response, with more than one third of patients achieving 2 year clinical remission and more than $40 \%$ persisting on treatment after 5 years. Unexpectedly, no significant difference was found between first and second line of treatment. The use of CZP in childbearing women seems to be associated with a higher retention rate. Disclosure of Interest: None declared DOI: 10.1136/annrheumdis-2018-eular.4160

\section{FRI0115 INDIRECT STANDARDISED ASSESSMENT OF INJECTION SITE PAIN FOLLOWING SUBCUTANEOUS ADMINISTRATION OF CITRATE-FREE FORMULATION OF ADALIMUMAB AND ITS BIOSIMILAR ABP 501}

E. Krishnan, N. Zhang, H. Wang, R. Kaushik. Biosimilars Development, Amgen Inc., Thousand Oaks, USA

Background: In randomised double-blind studies in patients with rheumatoid arthritis (RA) and psoriasis (PsO), ABP 501, an approved adalimumab biosimilar had significantly lower injection site pain (ISP) perception compared with the citrate-containing formulation (CCF) of adalimumab reference product (RP) $(40 \mathrm{mg}$ $0.8 \mathrm{~mL}$ ); however, there have been no direct or indirect comparisons of ISP perception between ABP 501 and the CFF of the RP $(40 \mathrm{mg} / 0.4 \mathrm{~mL})$

Objectives: To demonstrate that pain perception after injection of both the ABP 501 formulation and the CFF-RP formulation was lower than after CCF-RP.

Methods: We analysed ISP perception data after injection from two ABP 501 phase 3 studies, one in patients with RA (NCT01970475) and another in patients with PsO (NCT01970488) and 2 citrate-free RP formulation studies (NCT01561313, NCT01502423) where the "control" group received the CCF-RP. In all studies, patients were equally randomised between the 2 treatment arms. Patients were asked to rate the ISP perception on a visual analogue scale (VAS) on which the current pain level was marked from $0 \mathrm{~cm}$ (no pain) to $10 \mathrm{~cm}$ (wors possible pain). We calculated Cohen's d-statistic for difference in ISP perception with ABP 501 compared with CCF-RP for the 2 ABP 501 studies. Similar comparisons were performed between the ISP perception associated with CFF-RF and CCF-RF. These measures were subsequently compared in a descriptive manner. Results: Both ABP 501 and CFF-RF were associated with lower ISP perception after injection with maximum reduction observed immediately post-injection. The $95 \%$ confidence intervals of relative reduction in ISP perception for ABP 501 formulation and the CFF-RP (table 1) were provided descriptively. 
